Geburtshilfe

\section{Körperliche Fitness und Schwangerschaftsverlauf}

Baena-García L et al. Association of objectively measured physical fitness during pregnancy with maternal and neonatal outcomes. The GESTAFIT Project. PLoS One 2020; 15: e0229079. doi:10.1371/journal.pone.0229079

Baena-García L et al. Correction: Association of objectively measured physical fitness during pregnancy with maternal and neonatal outcomes. The GESTAFIT Project. PLoS One 2020; 15: e0231230. doi:10.1371/journal. pone. 0231230

Werdende Mütter mit einer unproblematischen Schwangerschaft sollten regelmäßig Sport treiben. Wie wirkt sich die maternale Fitness im frühen II. bzw. späten III. Trimenon auf den Schwangerschafts- und den Geburtsverlauf aus? Inwiefern beeinflusst sie den Geburtsmodus und welche Vor- oder Nachteile hat sie für das Kind? Mit diesen Fragen beschäftigte sich ein spanisches Forscherteam im Rahmen einer Longitudinalstudie.

Am sogenannten GESTAFIT-Projekt nahmen 159 Schwangere teil. Sie absolvierten - je nach persönlicher Präferenz - entweder ab der 17. Gestationswoche bis zur Entbindung unter Anleitung ein aerobes Ausdauer- und Krafttraining oder bildeten die Kontrollgruppe. Sowohl nach etwa 16 als auch nach etwa 34 Schwangerschaftswochen erhoben die Wissenschaftler soziodemografische und anthropometrische Daten der Schwangeren, erfassten ihre gynäkologisch-geburtshilfliche Anamnese und unterzogen die Frauen einem Fitnesstest. Hierbei objektivierten sie unter anderem die Muskelkraft der oberen und unteren Extremität, die Beweglichkeit sowie die kardiorespiratorische Leitungsfähigkeit (maximale Sauerstoffaufnahme während einer Laufbandbelastung). Anschließend prüften die Wissenschaftler, inwiefern sich die verschiedenen Fitnessparameter auf den Schwangerschaftsverlauf (Geburtsmodus, Gestationsalter bei Geburt, Nutzung der Epiduralanästhesie, Dauer der Geburtsphasen, Geschlecht, Gewicht und Apgar des
Neugeborenen) sowie das Ergebnis der Nabelschnur-Blutgasanalyse auswirkten.

\section{Ergebnisse}

$61 \%$ der Studienteilnehmerinnen waren Erstgebärende und rund $76 \%$ der Schwangerschaften endeten in einer Vaginalgeburt. Eine größere Oberkörper-Muskelkraft mit 16 Schwangerschaftswochen (SSW) korrelierte signifikant mit einem höheren Geburtsgewicht der Kinder. Die Kraft der unteren Extremität war dagegen weder mit dem maternalen noch dem neonatalen Outcome assoziiert. Es bestand ein signifikanter Zusammenhang zwischen einer höheren Beweglichkeit mit 16 SSW und einem stärker alkalischen arteriellen Nabelschnur$\mathrm{pH}$, einem höheren arteriellen Sauerstoffpartialdruck $\left(\mathrm{pO}_{2}\right)$, einer höheren arteriellen Sauerstoffsättigung sowie einem niedrigeren arteriellen $\mathrm{CO}_{2}$-Partialdruck $\left(\mathrm{pCO}_{2}\right)$. Eine bessere kardiorespiratorische Leistungsfähigkeit mit 16 SSW ging ebenfalls mit einem signifikant höheren arteriellen $\mathrm{pO}_{2}$ sowie einer höheren arteriellen Sauerstoffsättigung einher. Die körperliche Fitness mit 34 SSW korrelierte weder mit dem maternalen noch dem neonatalen Behandlungsergebnis. Die Analyse der Geburtsmodi ergab: Frauen, die per Kaiserschnitt entbunden wurden, hatten im Vergleich zu den Frauen mit einer Vaginalgeburt im II. Trimenon eine signifikant geringere kardiorespiratorische Leistungsfähigkeit und Beweglichkeit und wiesen sowohl mit 16 als auch mit 34 SSW eine signifikant geringere Gesamt-Fitness auf.

\section{FAZIT}

Werdende Mütter können vermutlich ihren Kindern einen besseren Start ins Leben ermöglichen und ihr Kaiserschnittrisiko senken, indem sie während der Schwangerschaft ihre körperliche Fitness steigern, meinen die Forscher. Weitere wissenschaftliche Untersuchungen müssen, ihrer Ansicht nach, diese Ergebnisse bestätigen.

Dr. med. Judith Lorenz, Künzell 